\title{
Jet Jumping: Low-Frequency Variability in the Southern Ocean
}

\author{
CHRISTOPHER C. CHAPMAN \\ Research School of Earth Sciences, and ARC Centre of Excellence for Climate System Science, The Australian National University, \\ Canberra, Australian Capital Territory, and CSIRO Wealth From Oceans Flagship, Hobart, Tasmania, Australia
}

\author{
ANDREw MCC. HOGG \\ Research School of Earth Sciences, and ARC Centre of Excellence for Climate System Science, The Australian National \\ University, Canberra, Australian Capital Territory, Australia
}

(Manuscript received 12 July 2012, in final form 17 December 2012)

\begin{abstract}
The authors study intrinsic variability in the position of jets in a $\beta$-plane channel ocean with simple topography using a quasigeostrophic numerical model. This study links the variability in jet position with abyssal anticyclones that form as a result of interaction of mesoscale eddies and subsurface topography, reminiscent of such flows as the Zapiola anticyclone. A simple dynamical framework explaining this behavior is developed. In this framework, this study shows that the topographic anticyclones form closed regions of homogenized yet time-varying potential vorticity. Neighboring topographic anticyclones are coupled by eddy fluxes. Interaction of a baroclinic jet with these two (or more) anticyclones can drive variability in local jet strength. Predictions of the dynamical framework are then compared with the results of the numerical model, and it is demonstrated that this model has merit in explaining the observed model variability. This study argues that this simple mode of variability has relevance for the ocean.
\end{abstract}

\section{Introduction}

\section{a. Background}

High-resolution satellite altimetry, long time hydrographic sections, and eddy-resolving numerical models have revealed that the Southern Ocean flow field is dominated by thin, strong, and quasi-zonal jetlike features. These jets show significant time variability: strengthening and weakening, splitting and merging, and shifting meridional position. However, the position of these jets is largely set by large, sub-surface topographic features (Rintoul et al. 2001). Observations reveal dramatically reduced temporal variability in the vicinity of these features (Sokolov and Rintoul 2009). This has led to the claim that jets are "locked in position and cannot shift" by these features (Sokolov and Rintoul 2009) and that the variability of frontal positions is much reduced in the vicinity of topography (Sallée et al. 2008). An

\footnotetext{
Corresponding author address: C. C. Chapman, Research School of Earth Sciences, The Australian National University, Canberra, ACT 0200, Australia.

E-mail: chris.chapman@anu.edu.au
}

explanation for this behavior is given in Vallis and Maltrud (1993).

Recent observations of the Southern Ocean obtained from satellite altimetry and fixed moorings have, however, indicated that jets will suddenly change preference of topographic feature. A jet that previously skirted a plateau to the north may very quickly (over time spans of days or weeks) shift to skirt the plateau to the south and obtain a new quasi-steady state. Shifts in the latitudinal positions of jets of more than $10^{\circ}$ have been observed in the vicinity of large topography such as the Kerguelen plateau (Sokolov and Rintoul 2009). Similar behavior was found in the idealized, quasigeostrophic simulations of Thompson (2010), which provides some evidence that the mechanism driving the variability in jet position is due to eddy-mean flow interaction. Fixed mooring measurements of the flow through gaps in the Macquarie Ridge south of New Zealand (Williams et al. 2010) show anticorrelated fluctuations through adjacent gaps. However, the hydrology indicates that fronts (at least as defined by hydrographic variables such as sharp temperature or salinity changes) only rarely change their preference of topographic feature. With doubt being cast on the global applicability of the methods of Sokolov 
and Rintoul (2009) (see Thompson et al. 2010) there is currently some confusion about whether a particular front is changing its position, or neighboring fronts are undergoing anticorrelated strengthening/weakening. Resolving this issue is beyond the scope of this paper and, as such, we argue that to both kinds of behavior may be defined as "jet jumping."

In a somewhat separate line of research, the generation of mean flows by eddy-topographic interaction has received attention in recent years, motivated by problems such as the Zapiola anticyclone, a topographically induced circulation in the South Atlantic associated with a particular seamount (Saunders and King 1995). These circulations have their origin in eddies interacting with topography at abyssal depths. A steady-state, quasibaroclinic theory for the development of closed circulations in the vicinity of closed geostrophic contours was put forth by Dewar (1998). In this theory, the balance between the eddy-advected potential vorticity and frictional effects determines the strength of the topographic circulation. While Dewar (1998) finds that these circulations are first order barotropic, more recent work suggest that they are bottom intensified in certain parameter regimes (Venaille 2012).

The time-dependent behavior of the Zapiola anticyclone was explored by Volkov and Fu (2008) who used a combination of satellite altimetry and output from the high resolution, data assimilating Estimating the Circulation and Climate of the Ocean, Phase II (ECCO2) model. They found that the strength of the anticyclone was temporally variable and that the variability was primarily influenced by the advection of vorticity over the seamount. Venaille et al. (2011) built upon this work, deriving a simple, barotropic model for the temporal variability of the anticyclone, in which the time-dependent variations in vortex strength were governed by fluxes of vorticity into the circulation region by eddy activity. The resulting red spectrum was compared to the output of eddy-permitting DRAKKAR (http://www.drakkar-ocean. eu) model and found to be in very good agreement.

In their paper, Venaille et al. (2011) state "one might conjecture that the mechanism described in this letter could play an signicant role in the internal low frequency dynamics and energetics of large scale oceanic currents (p. 5)." Here we show an example for which this conjecture turns out to be correct, positing that variability in these topographic circulations can interact with baroclinic, ACC-like jets to drive the "jet jumping" behavior described in Sokolov and Rintoul (2009). To investigate this phenomenon, we use an idealized, high-resolution, quasigeostrophic numerical model. We develop a timevarying framework for topographically generated circulations and their effect on jetlike flows originating upstream of the topographic feature in question. This model has its origins in the hypothesis of Hogg and Blundell (2006) to explain circumpolar variability in their quasigeostrophic model of the Antarctic Circumpolar Current.

The construction of the dynamical framework owes much to the various models to explain the maintenance of the general circulation (Andrews and McIntyre 1976) and annular mode-type variation of the extratropical atmosphere (Vallis et al. 2004). However, in contrast to the atmospheric studies, large topographic features dominate the background PV in the ocean, giving rise to nonzonal flows (Plumb and Ferrari 2005), complicating the geometry of the problem. However, the success of the Venaille et al. (2011) model provides compelling evidence that the physical mechanisms are very similar, even if the geometry differs.

In the next section, the variability of the ocean model is characterized. In section 3, we propose a framework of low-frequency variability of flows in the vicinity of topographic features and in section 4, we interpret the results of the numerical model in terms of the framework presented in section 3 .

\section{b. Ocean model}

For this paper, we use the ocean core of the quasigeostrophic coupled general circulation model (Q-GCM) as described in Hogg et al. (2003). This model time steps the quasigeostrophic potential vorticity equation in each layer $i \in[1, N]$ :

$$
\frac{\partial q_{i}}{\partial t}+J\left(\psi_{i}, q_{i}\right)=\mathcal{F}_{i}-\mathcal{D}_{i}
$$

and then corrects the streamfunction by inverting the elliptic equation:

$$
q_{i}=\nabla^{2} \psi_{i}+\mathbf{A}_{i j} \psi_{j}+\eta_{i},
$$

where suffix notation has been used to indicate the matrix-vector product. Here, $\eta$ is the time invariant background PV: $\eta_{i}=\beta y+\left(f_{0} / H_{N}\right) h(x, y) \delta_{i N}$, where $h$ is the topography, $\mathbf{A}_{i j}$ is the layer-coupling matrix, defined in Hogg et al. (2003), $\mathcal{F}_{i}, \mathcal{D}_{i}$ are the nonconservative forcing and dissipation terms, respectively, and $N$ is the number of layers. We take the forcing to be proportional to the wind stress curl in the upper layer and zero in all other layers, while the dissipation is taken to be biharmonic viscosity acting on vorticity $\left(\nu \nabla^{6} \psi\right)$ in all layers, with the addition of a linear Ekman drag $\left(-E \nabla^{2} \psi\right)$ in the lower layer. The model uses the RobertAsselin-Williams (RAW) filter (Williams 2009) in place of the standard Robert-Asselin filter in the time 
TABLE 1. Standard parameters for simulations.

\begin{tabular}{|c|c|c|}
\hline Parameters & Value & Description \\
\hline$f_{0}$ & $-1.2 \times 10^{-4} \mathrm{~s}^{-1}$ & Mean Coriolis parameter \\
\hline$\beta$ & $1.3 \times 10^{-11}(\mathrm{~m} \mathrm{~s})^{-1}$ & Coriolis parameter gradient \\
\hline$X \times Y$ & $1026 \mathrm{~km} \times 5000 \mathrm{~km}$ & Domain size \\
\hline$\Delta x, \Delta y$ & $5 \mathrm{~km}$ & Horizontal grid spacing \\
\hline$\Delta t$ & $75 \mathrm{~s}$ & Time step \\
\hline$\rho_{0}$ & $1000 \mathrm{~kg} \mathrm{~m}^{-3}$ & Reference density \\
\hline$\alpha$ & 5 & $\begin{array}{l}\text { Partial slip boundary } \\
\text { condition coefficient }\end{array}$ \\
\hline$H_{i}$ & $(300,1100,2600) \mathrm{m}$ & Layer thicknesses \\
\hline$g_{i}^{\prime}$ & $(5,2.5) \times 10^{-2} \mathrm{~m} \mathrm{~s}^{-2}$ & Reduced gravity \\
\hline$\nu$ & $1.0 \times 10^{10} \mathrm{~m}^{4} \mathrm{~s}^{-1}$ & $\begin{array}{l}\text { Biharmonic viscosity } \\
\text { coefficient }\end{array}$ \\
\hline$\delta_{\text {ek }}$ & $20 \mathrm{~m}$ & $\begin{array}{l}\text { Bottom Ekman-layer } \\
\text { thickness }\end{array}$ \\
\hline$W_{0}$ & $1.0^{-4} \mathrm{~m}^{2} \mathrm{~s}^{-2}$ & Maximum wind stress \\
\hline
\end{tabular}

stepping routine, ensuring formal second-order accuracy in time.

The model has three layers. The parameters used in this study are given in Table 1 . We remind the reader that in the Southern Hemisphere $f_{0}<0$. It is important to note that the horizontal grid spacing chosen for this study $(5 \mathrm{~km})$ is less than the deformation radius for the choices of Coriolis parameter and stratification $(\sim 10 \mathrm{~km})$, which in our experience permits adequate representation of the effect of eddies on the mean flow.

We use a zonally reentrant channel on a $\beta$ plane as an analog for the Southern Ocean. Forcing is provided by a zonally invariant westerly wind stress, with a truncated sinusoidal profile in the meridional direction:

$$
\tau_{0}(y)= \begin{cases}0 & y<L_{0} \\ W_{0} \cos \left(\frac{\pi y}{L_{w}}\right) & L_{0}<y<L_{y}-L_{0} . \\ 0 & y<L_{y}-L_{0}\end{cases}
$$

Here, $L_{y}$ is the meridional width of the domain, $L_{0}$ is the width of the region of no forcing, and $L_{w}=L_{y}-2 L_{0}$ (shown in Fig. 1). The peak wind stress $W_{0}$ is $1.0 \times$ $10^{-4} \mathrm{~N} \mathrm{~m}^{-2}$. This wind stress profile is chosen to mimic the wind stress exerted on the Southern Ocean by the midlatitude atmosphere. The regions of no forcing on
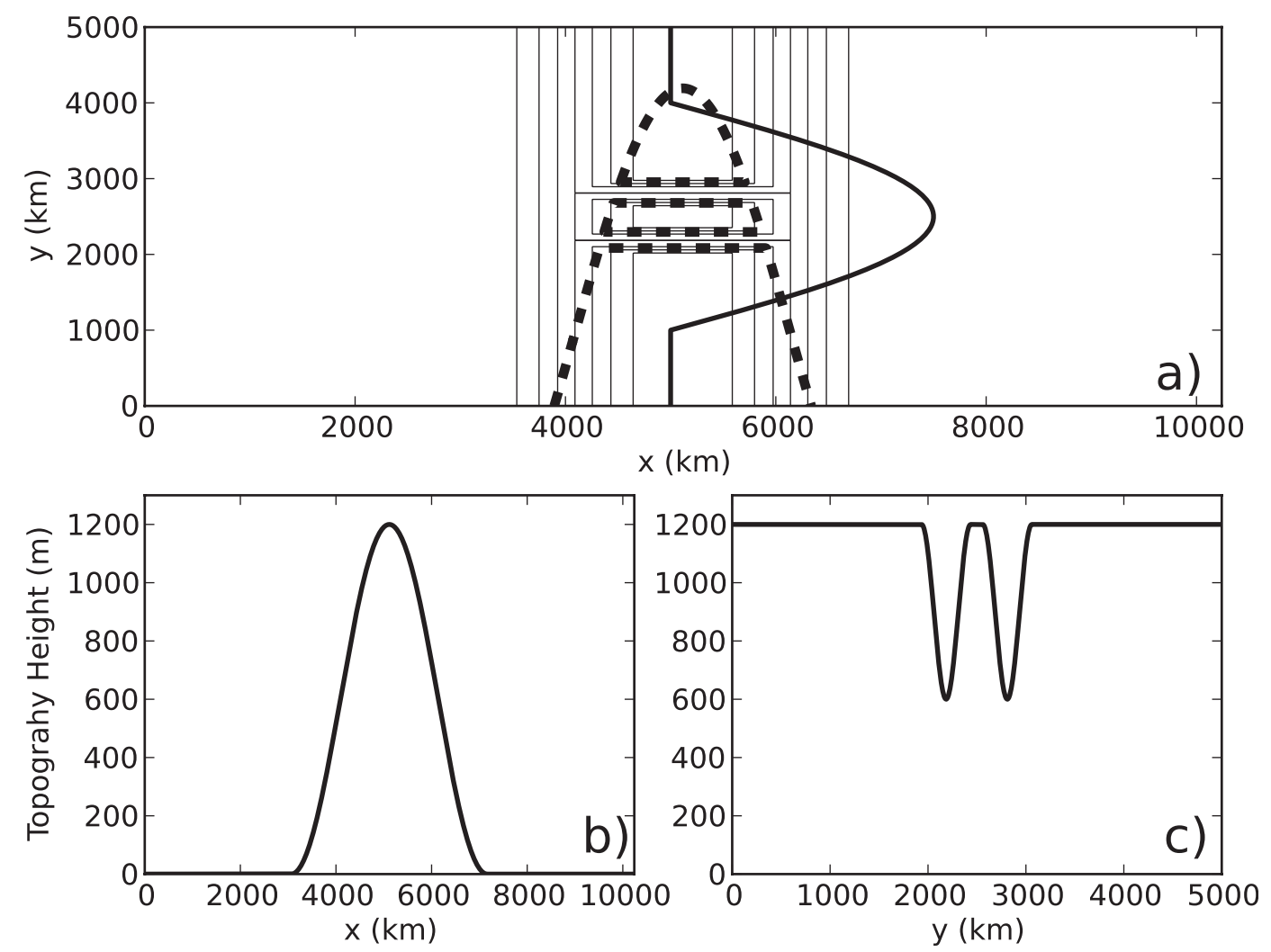

FIG. 1. (a) Domain layout showing topography (light) and wind stress (boldface) along with greatest closed $f / H$ contour (boldface, dashed); (b) zonal transect of topography, showing basic profile; (c) meridional transect of topography, showing canyon profile, written as $h_{0}(y)$ in the text. 

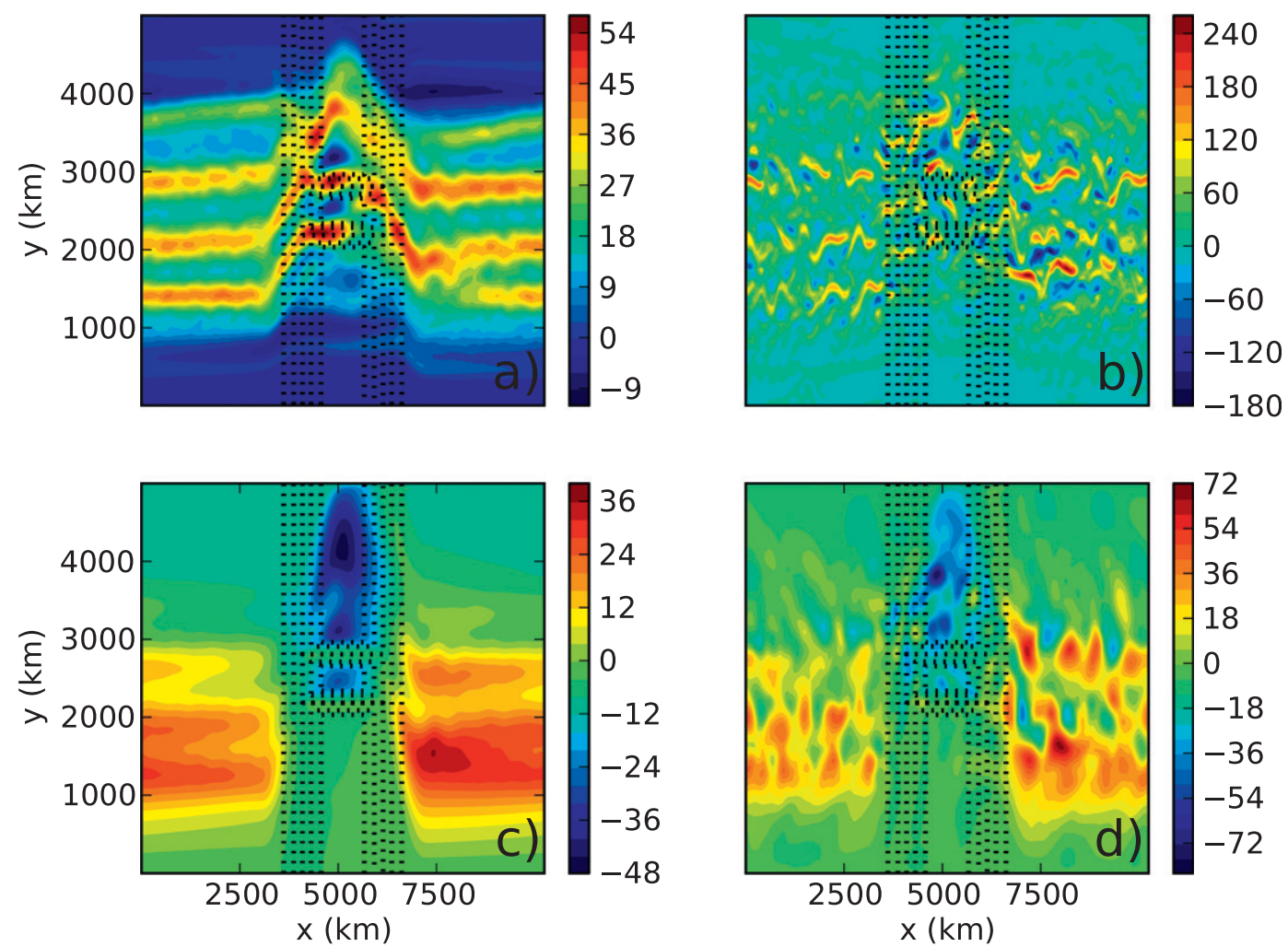

FIG. 2. The flow of the idealized numerical model. (a) Upper-layer temporal mean zonal velocity $\left\langle u_{1}\right\rangle\left(\mathrm{cm} \mathrm{s}^{-1}\right)$; and (b) snapshot of the upper-layer zonal velocity $u_{1}\left(\mathrm{~cm} \mathrm{~s}^{-1}\right)$. (c) Lower-layer temporal mean streamfunction $\left\langle\psi_{3}\right\rangle(\mathrm{Sv})$; and (d) snapshot of the lower-layer streamfunction $\psi_{3}(\mathrm{~Sv})$. (light, dotted lines show topographic contours).

either side of the sinusoidal profile are designed to remove influences from the edges of the channel.

Bottom topography takes the form of an isolated, meridional ridge, with two "canyons" or notches distributed about the center of the domain. The profile of the topography is given by

$$
h(x, y)= \begin{cases}0 & x<-2 L_{\mathrm{hw}} \\ h_{0}(y) \cos ^{2}\left(\frac{\pi}{4} L_{\mathrm{hw}} x\right) & -2 L_{\mathrm{hw}}<x<2 L_{\mathrm{hw}} \\ 0 & y<2 L_{\mathrm{hw}}\end{cases}
$$

Here, $L_{\mathrm{hw}}$ is the hill half-length, and $h_{0}(y)$ is the meridionally varying part of the topography. The height of the topography is set to be $45 \%$ of the lower-layer depth. The profile of the "canyons," encapsulated in $h_{0}(y)$ is a simple, half-period cosine function. This topography and its spatial relation to the wind stress are shown in Fig. 1.

The canyons are approximately $200 \mathrm{~km}$ wide, separated by a gap of approximately $150 \mathrm{~km}$, chosen to ensure that the canyons themselves and the gaps between them span multiple eddy length scales, and therefore, variability in the vicinity of these features are dominated by large-scale effects. The height of the topography is set such that regions of closed $f / H$ contours (blocked geostrophic contours) are formed, which is crucial for the development of jet-jumping variability. These regions are indicated in Fig. 1a.

The model is spun up from rest until statistical equilibrium, which takes approximately 70 years. The model is then run for an additional 90 years, which returns enough data to adequately investigate the variability.

\section{Low-frequency variability}

\section{a. Mean flow}

The time mean zonal velocity in the upper layer (Fig. 2a) indicates a general eastward current organized into three or four primary jets. These jets deviate strongly from the zonal direction in the vicinity of the topography, making meridional detours to flow "through" the canyon regions. The mean streamfunction in the lower layer (Fig. 2c) shows two anticyclones that have formed on either side of the northern canyon (streamfunction is chosen over velocity as it facilitates the visualization of 

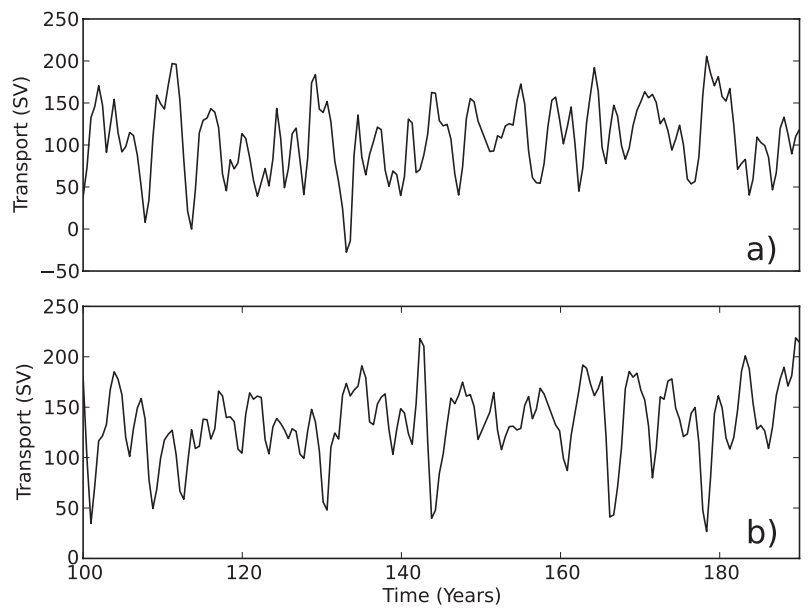

FIG. 3. Barotropic transport "through" the (a) northern canyon and (b) southern canyon. Calculations are performed at the domain midpoint.

the anticyclones). This flow field is predicted by the theory of Dewar (1998). The jet structure in the surface layer is not apparent in the lower layers. The time mean of the barotropic streamfunction (not shown) indicates that the influence of the topographic vortices extends throughout the depth of the fluid, consistent with the results of Dewar (1998). Snapshots of the flow field in the upper layer (Fig. $2 \mathrm{~b}$, zonal velocity) and lower layer (Fig, 2d, streamfunction) show a well-developed eddy field in both layers.

\section{b. Temporal variability}

\section{1) UPPER-LAYER TRANSPORT}

As this paper is concerned primarily with local variability in jets, an obvious metric to use is zonal transport. As such, we compute the total transport "through" each canyon at the zonal midpoint of the domain and then apply a low-pass filter (a finite-impulse response Lanczos filter) to the resulting time series. ${ }^{1}$ The two transport time series are shown in Fig. 3.

The transport time series are highly variable and show substantial anticorrelation: an empirical orthogonal function (EOF) analysis of the two time series reveals that the anticorrelated mode explains $\sim 65 \%$ of the variance. ${ }^{2}$ The transport time series are also quasiperiodic, with a period of approximately 5 years.

\footnotetext{
${ }^{1}$ Since the model is quasigeostrophic (QG), there can technically be no transport "through" these canyons. The canyons only provide a local variation in the background PV. More correctly, we should state that we compute the transport "over" the canyons.

${ }^{2}$ An EOF analysis of a two dimensional dataset will decompose it into a correlated mode and an anticorrelated mode (Wilks 2006).
}

The transport variability through both canyons is high. Through the northern canyon (Fig. 3a), transport varies between maximum values of 203 Sverdrups ( Sv; $1 \mathrm{~Sv} \equiv$ $10^{6} \mathrm{~m}^{3} \mathrm{~s}^{-1}$ ) to a minimum values of $-26 \mathrm{~Sv}$, indicating reverse (westward) flow at times. The mean transport is $96 \mathrm{~Sv}$, with a standard deviation of $12.3 \mathrm{~Sv}$. The southern canyon transport varies between 30 and $220 \mathrm{~Sv}$, with a mean of $114 \mathrm{~Sv}$ and a standard deviation of $11.9 \mathrm{~Sv}$.

\section{2) SPATIAL PATTERNS OF VARIABILITY}

To further investigate the variability of the system, we define the "large-scale" flow, or the filtered field, as $\langle\psi\rangle$ and the "eddy field," as $\psi^{\prime}$, such that the field is decomposed as

$$
\psi_{i}(\mathbf{x}, t)=\left\langle\psi_{i}\right\rangle+\psi_{i}^{\prime},
$$

with $\left\langle\psi^{\prime}\right\rangle=0$.

We first low-pass filter the velocity streamfunction field (as before, using a Lanczos filter with a 6 month cutoff period) and then subsample at twice the Nyquist frequency $\left(8 \mathrm{yr}^{-1}\right)$. A standard EOF analysis (Wilks 2006) is then performed on the low-pass-filtered data. In performing this EOF, we restrict attention to the portion of the domain in the vicinity of the topography and the canyons, to remove the influence of the variability in the regions away from the region of interest. The EOF analysis is performed independently in each layer. Performing the EOF analysis on all three layers simultaneously does not appreciably change the results.

The first EOF of the upper (lower) layer is shown in Figs. 4a,b. The eigenvalue spectrum of the EOF analysis indicates that the first EOF is the dominant mode of variability, explaining $\sim 15 \%$ of the variance in the upper layer and $\sim 22 \%$ of the variance in the lower layer. The second EOF, by contrast, explains $\sim 5 \%$ of the variance in each layer. Investigations of the next five EOFs indicate little coherent structure. We thus use the first EOF and its associated principal component (PC) time series as a metric of the low-frequency variability.

The first EOF shows a dipolar structure in the lower layer, with poles on either side of the northern canyon. This pattern describes an anticorrelated strengthening (weakening) of the northern (southern) topographic anticyclones. The EOF of the upper layer shows a tripolar structure. There is clearly a strong barotropic component to this structure: with the exception of the weaker southern pole, the upper-layer and lower-layer EOF structure are qualitatively similar. The PC time series of this EOF (Fig. 5) shows a quasi-periodic signal with a period of approximately 5 years.

To understand how this variability manifests, we define the "key states" of the low-frequency mode (Berloff 

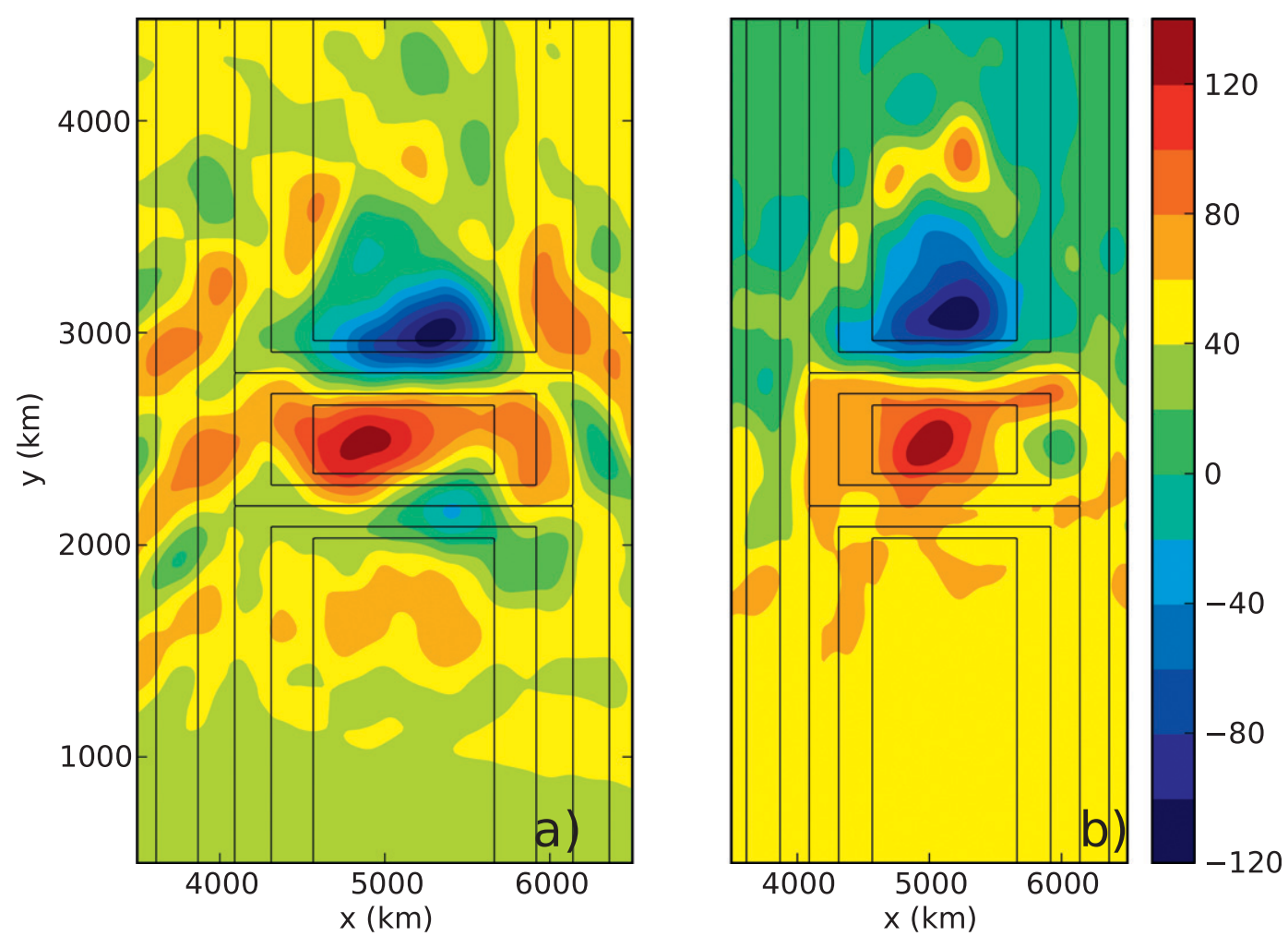

FIG. 4. First EOFs of the streamfunction on a truncated domain (a) upper layer and (b) lower layer (light, solid lines show topography).

et al. 2007). We do this by employing a conditionalensemble mean as a low-pass filter. We define, somewhat arbitrarily, that the system exists in key state "A" when the PC time series (normalized by standard deviation) of the first EOF has a value greater than, or equal to, 0.6. Likewise, key state " $\mathrm{B}$ " is defined as the times when the PC time series is less than, or equal to, -0.6 . We then average all time steps in each state to form the conditional ensemble means: $\langle\psi\rangle^{A}$ and $\langle\psi\rangle^{B}$. The system exists in state $A \sim 26 \%$ and state $B \sim 32 \%$ of the time. The anomalies of these states defined relative to the time mean: (i.e., $\langle\psi\rangle^{K}-\langle\psi\rangle$ ) are presented in Fig. 6.

State A corresponds to a stronger jet associated with the northern canyon and a weaker jet associated with the southern canyon (relative to the time mean), shown in Figs. 6a,b. State A shows a substantial $(\sim 30 \%)$ increase in the zonal velocity associated with the jet in the northern canyon and a corresponding decrease $(\sim 20 \%)$ in the zonal velocity of the jet in the southern canyon. State B shows the reverse situation to state A: a $\sim 30 \%$ reduction in the zonal velocity in the northern canyon, along with a $\sim 15 \%$ increase in the southern canyon.

We also note several differences in the streamfunction fields in the lower layer between state A and B (Fig. $6 \mathrm{c}, \mathrm{d}) .\langle\psi\rangle^{A}$ shows a substantially stronger topographic anticyclone to the north of the northern canyon, while $\langle\psi\rangle^{B}$ shows this anticyclone to be substantially weaker. The anticyclone in the center of the domain remains mostly unchanged in both states A and B.

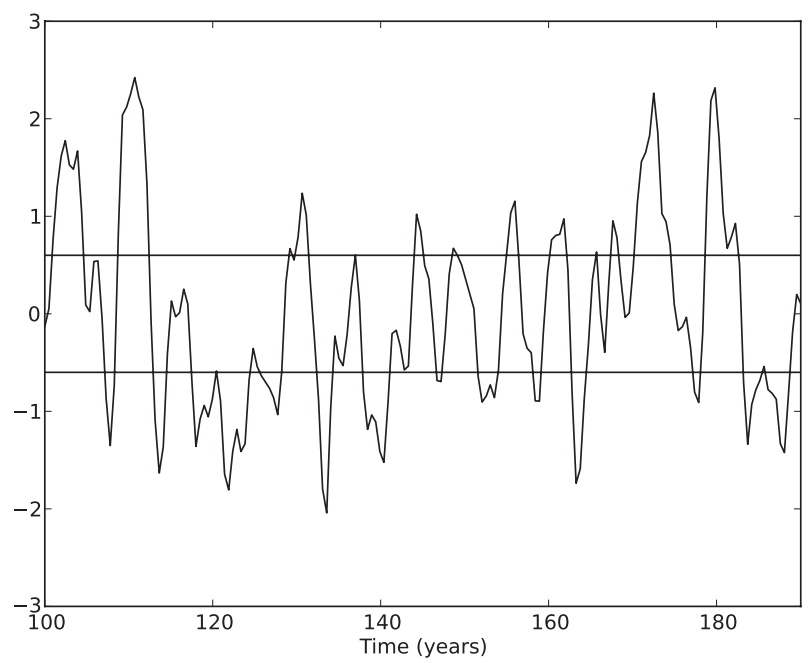

FIG. 5. Principle component time series for the first EOF, normalized by variance. The horizontal lines indicate the thresholds for key states (top) A and (bottom) B. 

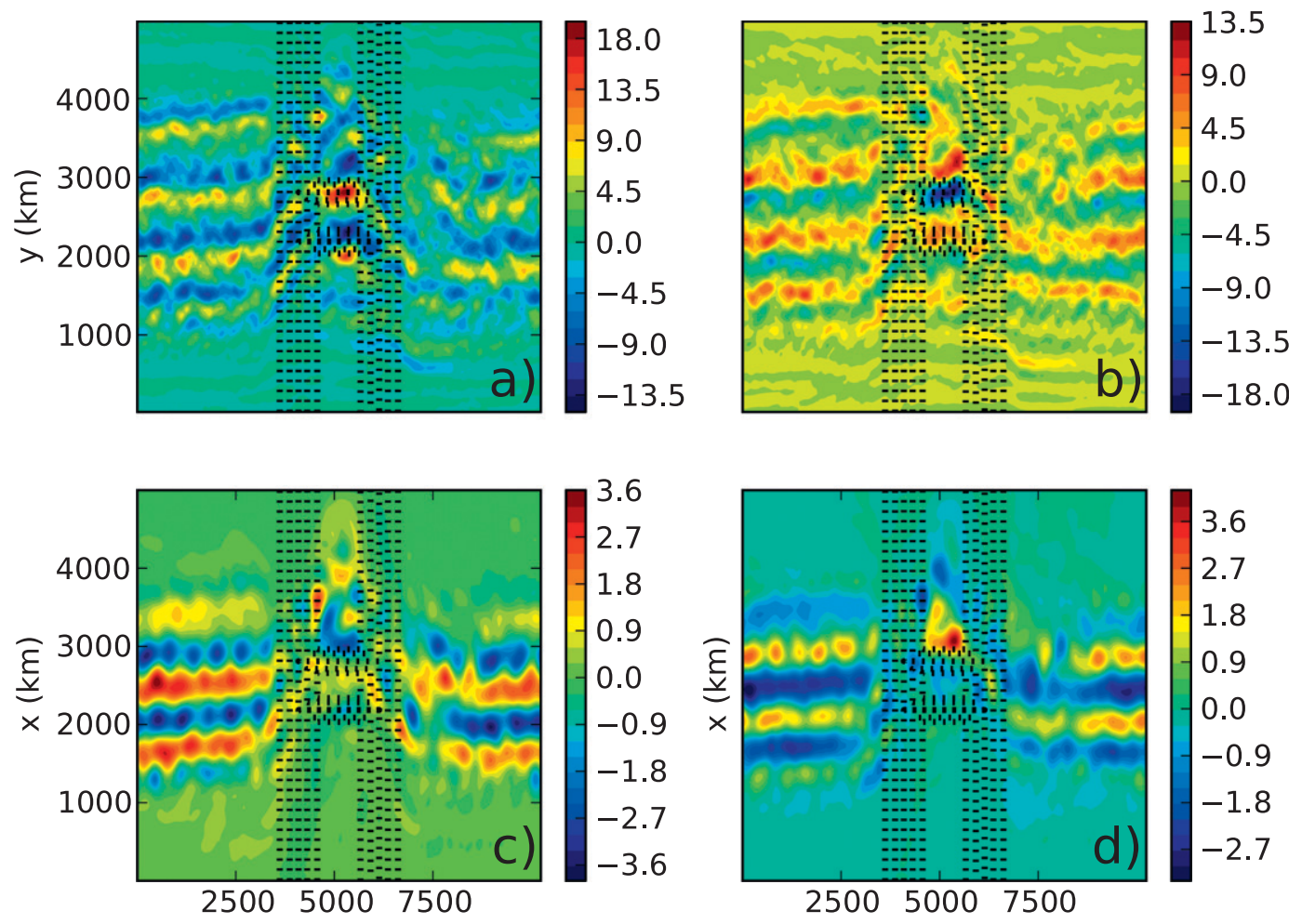

FIG. 6. Key state anomalies (difference between the ensemble mean and the temporal mean): (a) state A upperlayer zonal velocity anomaly $\left(\mathrm{cm} \mathrm{s}^{-1}\right)\left\langle u_{1}\right\rangle^{A}-\left\langle u_{1}\right\rangle$; (b) state B upper-layer zonal velocity anomaly $\left(\mathrm{cm} \mathrm{s}^{-1}\right)\left\langle u_{1}\right\rangle^{B}-$ $\left\langle u_{1}\right\rangle$; (c) state A lower-layer streamfunction anomaly (Sv) $\left\langle\psi_{3}\right\rangle^{A}-\left\langle\psi_{3}\right\rangle$; and (d) state B lower-layer streamfunction anomaly (Sv) $\left\langle\psi_{3}\right\rangle^{B}-\left\langle\psi_{3}\right\rangle$.

\section{c. Relationship of spatial patterns to other metrics of temporal variability}

In Fig. 7 the PC time series of the first EOF is plotted with the upper-layer transport time series from the southern canyon (both time series are centered and normalized by standard deviation) computed in section $2 \mathrm{a}$. The two time series are obviously highly correlated, implying that the first EOF of the upper layer is describing the same variability as the transport time series.

The relative vorticity in the lower layer, $\zeta_{3}=\nabla^{2} \psi_{3}$, is integrated over a contour of $f / H$ that encloses each topographic anticyclone. As with the transport time series, we compare this metric to the PC time series for the first EOF. Once again, the time series (not shown) are strongly correlated $\left(R^{2}=0.714\right)$, implying that the variability described by the lower-layer EOF and the integrated $\zeta_{3}$ are similar processes.

Thus, in terms of the EOFs and the key states, we can characterize the variability either in terms of coupled strengthening/weakening of surface jets or, in terms of the strengthening/weakening of the northern topographic anticyclone. Both of these phenomena are described by the first EOF, with an associated quasi-periodic PC time series. We elucidate the dynamical link between the jet transport and topographic anticyclone in the next two sections.

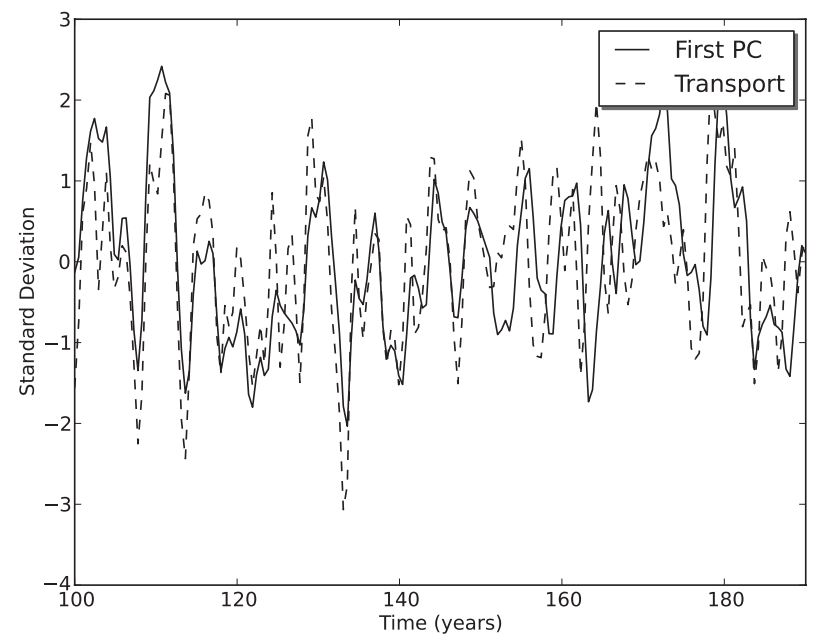

FIG. 7. Barotropic layer transport "through" the southern canyon (dashed), plotted with the first PC time series of the upper layer (solid). 


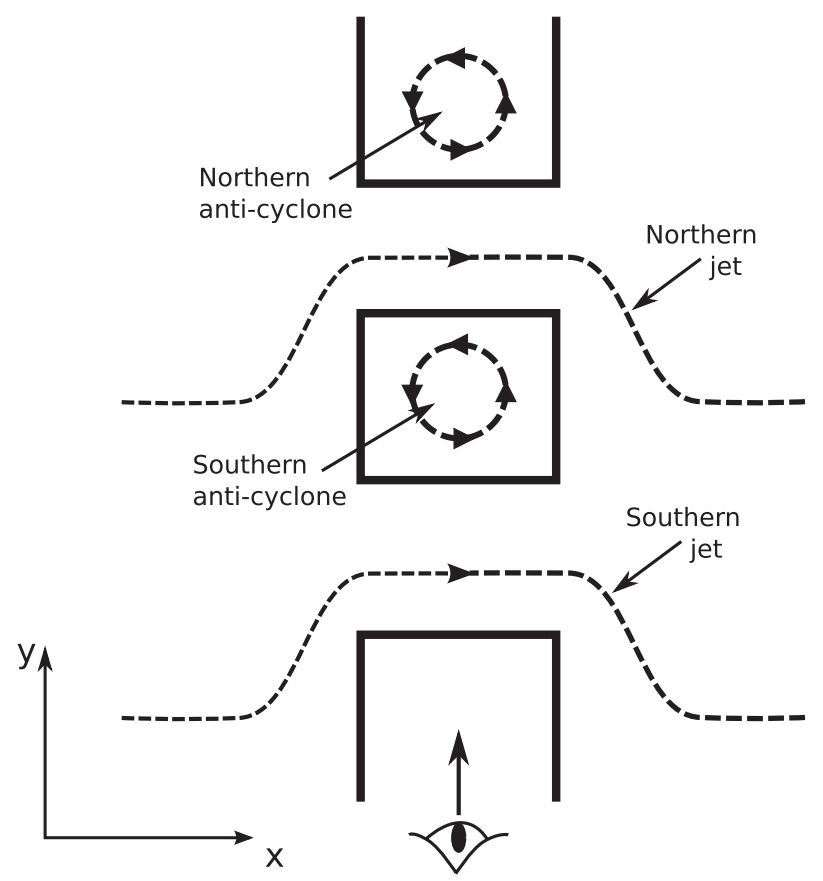

FIG. 8. Schematic of the system showing the two jets and two topographic anticyclones. The eye indicates the location of the transect shown in Fig. 9.

\section{Dynamics of the low-frequency variability}

In this section, we describe the dynamics of the lowfrequency variability using a simple framework. This framework is similar to that used in studies of maintenance and vacillation of the westerly jet in the midlatitude troposphere or tropical stratosphere, such as Andrews and McIntyre (1976).

\section{A simple mechanism of the low-frequency variability}

In Fig. 8 we sketch a schematic of the mean state of the flow as described in section 2. This diagram shows the system reduced to four essential components: two barotropic anticyclones in the regions of blocking topography, and two equivalent-barotropic jets that are aligned with the canyons.

In section 2 we noted that the dominant mode of variability is primarily barotropic and that the jets are approximately equivalent barotropic. In this section, we assume a barotropic flow, and use the quasigeostrophic $\mathrm{PV}$ equation, written in the flux form:

$$
\frac{\partial q}{\partial t}+\nabla \cdot(q \mathbf{u})=\mathcal{F}-E \nabla^{2} \psi
$$

where the PV $q$, is given by

$$
q=\nabla^{2} \psi(x, y)+f(y)+\frac{f_{0}}{H_{0}} h(x, y),
$$

having made the rigid-lid approximation. Here, $H_{0}$ is the unperturbed depth of the fluid. Dissipation is provided by a bottom Ekman layer. As noted in section 2 and in Dewar (1998), in regions of blocked geostrophic contours, the flow is able to form closed pools of PV under certain conditions.

We assume that such pools exist in a region bounded by a closed geostrophic contour that defines the edge of a region $\Omega$. This contour, denoted $\partial \Omega$, is defined to be the largest closed $f / H$ contour that encompasses the topographic circulation. According to the theory of Dewar (1998), when there is motion in the upper layers, the closed PV contours are not exactly coincident with the geostrophic contours, with the former being shifted to the north and west. However, our numerical results, along with those of Dewar (1998) indicate that this shift is generally small, and that the closed PV contours can be taken as approximately coincident with the geostrophic contours. As such, $\partial \Omega$ is approximately the boundary of the topographic anticyclone and is considered time independent. When the anticyclone strength fluctuates, we consider only changes in the flow and not changes in the shape of the $\partial \Omega$.

Integrating Eq. (5) over $\Omega$ and employing the divergence theorem:

$$
\frac{\partial Q}{\partial t}+\oint_{\partial \Omega} q \mathbf{u} \cdot \mathbf{n} d \ell=-E \Gamma+\int_{\Omega} \mathcal{F} d A
$$

where uppercase quantities refer to the area-integrated results of their lowercase equivalents. The spatially integrated PV can be directly related to the circulation $\left(\Gamma=\int \zeta d A=\oint \mathbf{u} \cdot d \ell\right)$ of the closed topographic circulation by

$$
\Gamma=Q-\frac{f_{0}}{H_{0}} H-\int_{\Omega} f(y) d A
$$

In the current context the circulation of the topographic anticyclone is a measure of its "strength" in a spatially averaged sense. This expression relates the "strength" of the topographic anticyclone to the spatially averaged $\mathrm{PV}$.

Together these expressions describe the variability of a topographic circulation as a balance between the flux of PV through $\partial \Omega$ and the dissipation within $\Omega$. A similar expression was derived by Venaille et al. (2011) for radial geometry (although it should be noted that in their formulation, mass transport was used as a measure of anticyclone strength as opposed to circulation). The current 
approach is not restricted to any particular geometry and relies only on the existence of closed pools of PV.

As indicated in Fig. 2c, there are two topographic anticyclones in our idealized numerical model. We note that in the numerical experiments, the two topographic anticyclones lie adjacent to each other. Closer inspection reveals the a contour of $f / H$ divides the two circulations, which can be seen in Figs. $2 c$ and $4 a, b$. This contour acts as a northern (southern) edge of the southern (northern) anticyclone and lies very close to the axis of the northern most jet. As such, the two anticyclones share a portion of the $\partial \Omega$ curve that is taken to define the integration region.

This definition allows us to decompose the total PV flux into each anticyclone: the flux through the shared portion of $\partial \Omega$ plus the flux through the remainder. We note that the flux out of one closed circulation through the shared portion is equal to the flux into the other closed circulation. This expression couples the circulations in a manner suggestive of anticorrelated behavior. These PV fluxes include both mean flow and eddying components.

Assume two closed, barotropic anticyclones aligned meridionally. We denote the northern (southern) circulation with the superscript $N(S)$. Each circulation is bounded by a contour that forms a region $\Omega^{K}$ for $K \in[N, S]$. These contours are, in turn, decomposed into a shared $\partial \Omega_{s}^{K}$ and the remainder $\partial \Omega_{r}^{K}$. Applying Eq. (6) to each region, together with the definition of integrated $\mathrm{PV}$ in Eq. (7), we obtain the following:

$$
\begin{aligned}
& \frac{\partial Q^{N}}{\partial t}=-F_{s}^{N}-F_{r}^{N}-E \Gamma^{N}+\int_{\Omega^{N}} \mathcal{F} d A, \text { and } \\
& \frac{\partial Q^{S}}{\partial t}=F_{s}^{N}-F_{r}^{S}-E \Gamma^{S}+\int_{\Omega^{S}} \mathcal{F} d A,
\end{aligned}
$$

which defines the time-dependent strength of the circulation. In the above expressions, we have used the shorthand

$$
F_{s}^{K}=\oint_{\partial \Omega_{s}^{K}} q \mathbf{u} \cdot \mathbf{n} d \ell
$$

to refer to the PV fluxes through the shared contour, with a similar expression for the fluxes through the remainder of the contour.

The physics encompassed in these expressions is relatively straightforward. The circulation is described as a balance between the PV flux into the circulation regions and the dissipation of vorticity by the bottom Ekman layer. PV can be introduced by Ekman pumping owing to the wind forcing or by advection of PV by the

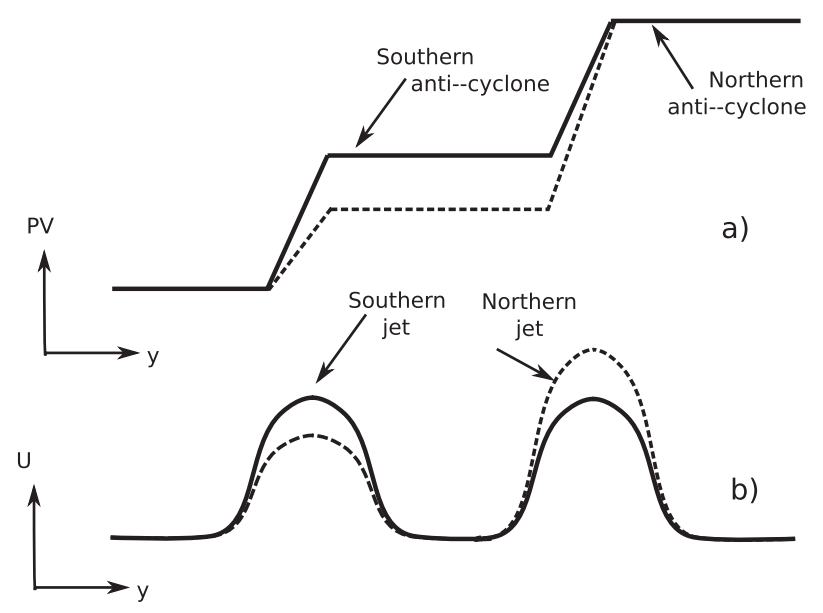

FIG. 9. Schematic of the system showing meridional transects of (a) PV and (b) zonal velocity. The solid lines indicate the mean system state with jets of roughly equal strength, while the dashed lines are indicative of the state in system A, with a stronger northern jet and a weaker southern jet.

flow (either mean flow or eddying). Similarly, PV can be advected out of the circulation region. Net PV gain within the circulation regions results in an increase in strength of the topographic circulation. This is simply a restatement of Kelvin's circulation theorem.

We now link the behavior of the closed topographic circulations to the behavior of the jets. Recall the "PV staircase" interpretation of zonal jets (Dritschel and McIntyre 2008), in which a jet in the velocity field is related to a step or otherwise rapid change in PV field by way of the PV invertability principle. The regions of weak flow in between the stronger jets are related to regions of more or less homogenous PV. Numerical results described in section 2 are suggestive of a staircaselike PV field, where the homogenized regions (the steps) are associated with the closed topographic circulations described above. A meridional transect of the PV field is sketched in Fig. 9a for both the temporal mean of the system and for a state resembling key state A.

Idealized computations (e.g., those of Dunkerton and Scott 2008) indicate that the strength of a jet associated with a particular PV "step" is proportional to the difference in PV between adjacent homogenised regions. In an equivalent barotropic ocean we can express the transport through the northern most canyon without reference to an imposed PV profile simply by

$$
T^{N}=C L_{D}\left(Q^{N}-Q^{S}\right),
$$

where $C$ is a constant, and $L_{D}$ is the deformation radius, with a similar expression for the transport through the southernmost canyon. This mechanism is sketched in 

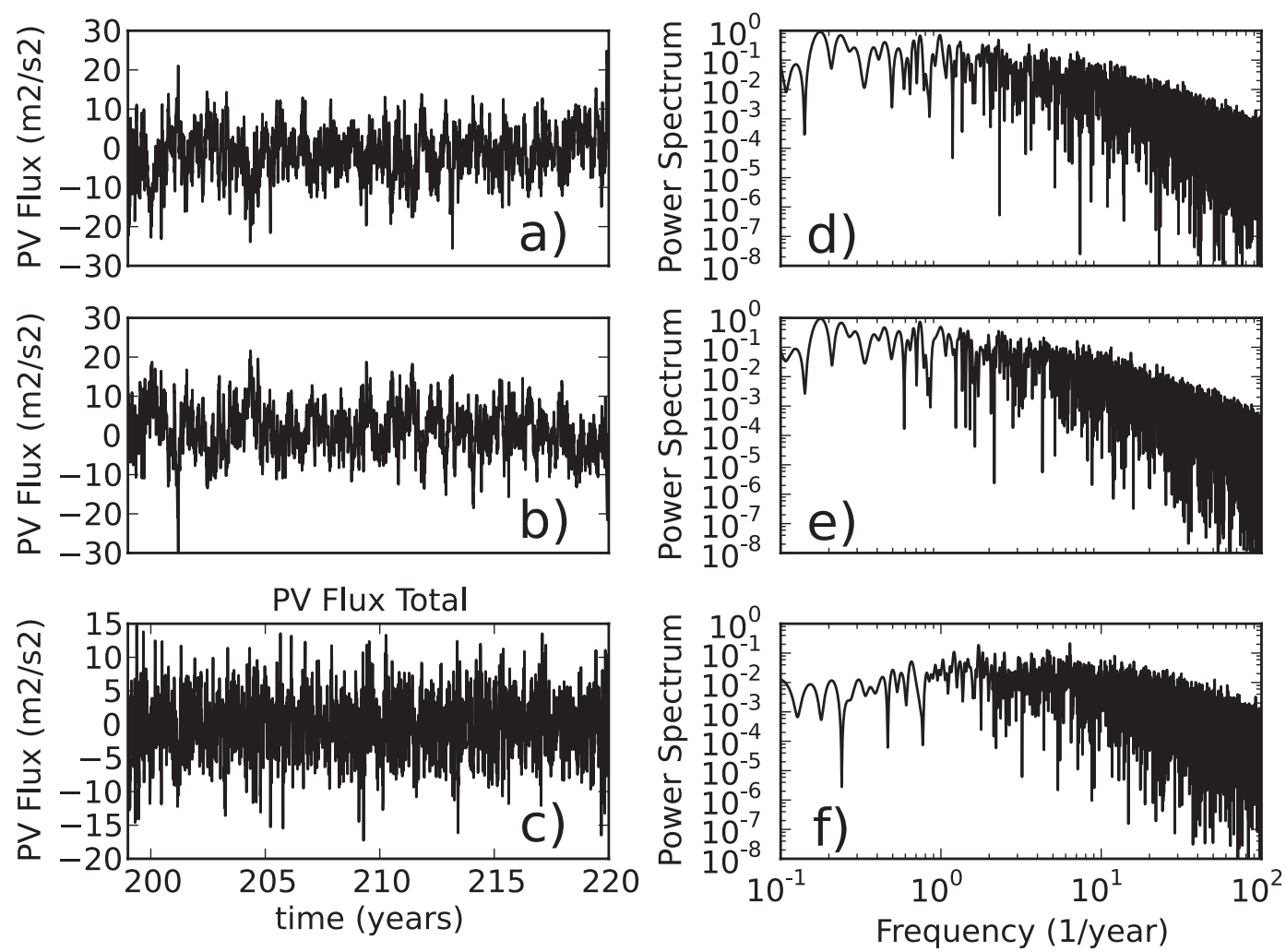

FIG. 10. Barotropic potential vorticity flux (left) time series and (right) spectra. (a) Time series of flux through the shared contour; (b) the time series through the remainder of the contour enclosing the circulation; and (c) is the time series of the total flux. (d) Power spectrum of flux through the shared contour; (e) power spectrum of flux through the remainder of the contour enclosing the circulation; and (f) power spectrum of the total flux.

Figs. 9a,b. The solid lines in Fig. 9a indicate a situation similar to the temporal mean, where $\Delta Q^{N} \approx \Delta Q^{S}$. This is reflected in the zonal velocity field (Fig. 9b, solid line) showing jets of approximately equal strength in the northern and southern canyons. The dashed lines in Fig. 9a represent a situation such as key state $\mathrm{A}$, where $\Delta Q^{N}>\Delta Q^{S}$. The zonal velocity field (Fig. 9b, dashed line) reflects this variation with a weaker southern jet and a correspondingly stronger northern jet.

We conjecture that the dynamics described in this section can give rise to the low-frequency variability described in section 2. In particular, we propose that the variability is driven by three key features:

(i) variability of the topographic circulations is driven by eddy fluxes and damped by friction;

(ii) variability between topographic circulations are coupled by eddy fluxes between each individual circulation; and

(iii) transport variability is described by the variation in the difference of the averaged PV of adjacent topographic circulations.
Each of these points will be validated using the results of our numerical simulation in section 4 .

\section{Variability in the numerical model}

The framework developed in section 3 allows a number of predictions that can be tested against the numerical model results presented in section 2 .

\section{a. Variability of topographic circulations}

The variability of the topographic circulations is expressed in Eqs. (8) and (9). The key driver of variability in these circulations is the flux of PV into the closed PV contours. Venaille et al. (2011) approximated this forcing term as stochastic with a white spectrum plus a constant term. In contrast, we compute the 20 years of barotropic PV flux directly from numerical model at each time step, which is accumulated and stored as a daily average, which ensures accurate representation of the eddy statistics (shown in Figs. 10a-c). The spectra of the PV flux (Figs. 10d-f) indicate flat power spectral density at low frequencies, with a power-law roll off only 
at high frequencies. This provides evidence that the PV flux spectra are approximately white and that the lowfrequency variability that manifests in the system is not a direct response to some frequency intrinsic in the forcing.

Using forcing taken from the numerical model, Eq. (9) is then solved using a third-order Adams-Bashforth scheme (initial conditions are taken directly from the numerical model). The solution is compared with $Q^{S}$ computed directly from model output. Point errors are generally limited to $5 \%$ or less of the standard deviation and the mean-squared error is $\sim 3 \%$. With the forcing term on the right-hand side of Eq. (9) taken directly from the numerical model output, this low error is not surprising. However, it does support the hypothesis that the PV flux driving mechanism of topographic circulation variability is operating in our model. It also provides strong evidence that the effects of wind and viscosity are small, as both are absent in our integrations and yet the error term is very small.

As described in Eqs. (8) and (9) eddy fluxes from two different sources drive the variability in the anticyclones: a common flux (through $\partial \Omega_{\text {shared }}$ ) and an external flux (through $\partial \Omega_{\text {remainder }}$ ). The common flux couples the two anticyclones, while the external flux is independent to each individual circulation. To validate the hypothesis that a flux of PV from one anticyclone to the other is responsible for their observed anticorrelation, we first assess the magnitudes of the forcing arising from each flux, shown in Figs. 10a,b for the southern anticyclone (similar results for the northern anticyclone are not shown). We see that the magnitudes of forcing common and external fluxes are approximately equal, and thus that common fluxes (that couple the circulations) are as important as external fluxes.

Does the existence of the common flux give rise to the observed anticorrelation? To test this, we again solve (9) using fluxes taken directly from the numerical simulation. However, we suppress the external flux term (i.e., we set $F_{r}^{S}=0$ ), and instead of comparing the solution to $Q^{S}$ as before, we instead normalize the result and compare it to the PC time series from section $2 b(2)$ that represents the anticorrelated mode of variability. Despite the external fluxes being suppressed, we still produce an output time series that is highly (but not perfectly) correlated with the PC time series (Pearson $r$ of 0.627 and coefficient of determination $R^{2}$ of 0.646$)$. We thus argue that the anticorrelated strengthening/ weakening of the anticyclone is driven predominantly by intergyre PV flux.

\section{b. Variability of jet transport}

According to our proposed dynamical mechanism, the variability of transport through the canyons is linked to the variability of topographic anticyclones by way of the PV staircase, described by Eq. (10). Here we test this assertion.

Over the complete 90 -yr simulation, we compute the area-integrated barotropic PV for both the northern $\left(Q^{N}\right)$ and southern $\left(Q^{S}\right)$ topographic circulations along with the PV integrated over the homogenized region south of the southernmost canyon. The (low-pass filtered) PV of this region is approximately constant over the period of the simulation and shall be referred to as $Q^{0}$. In addition, we compute the surface-layer transport through the northern and southern canyons over the entire simulation period (we have used surface layer, as opposed to barotropic transport, to better capture the surface-intensified jet dynamics). All quantities are lowpass filtered using a finite impulse response Lanczos filter with a cutoff period of 6 months.

According to our proposed mechanism, the transport through the northern canyon is correlated with the PV difference between the northern and southern topographic circulations:

$$
T^{N} \propto L_{D}\left(Q^{N}-Q^{S}\right),
$$

while transport through the southernmost canyon is proportional to the difference between the southernmost topographic circulation and the homogenized PV region to the south:

$$
T^{S} \propto L_{D}\left(Q^{S}-Q^{0}\right) .
$$

We assess formally the correlation between $\Delta Q$ and $T$ using a Pearson $r$ coefficient and the coefficient of determination $\left(R^{2}\right)$. For the northern canyon, the Pearson $r$ is 0.691 , while for the southern canyon, it is 0.664 . The coefficient of determination for the northern canyon is 0.708 while slightly higher for the southern canyon at 0.711 . The regression is statistically significant and as there is only a single free parameter there is only a small chance of overfitting. As such, the reported Pearson $r$ and coefficient of determination should be sufficient to assess the model's validity.

\section{c. Key-state analysis}

Finally, we reinterpret the key state analysis described in section 2 to describe the "jet jumping" behavior in light of our dynamic mechanism. Here, we tie together the various threads to show the value of this mechanism in explaining the observed variability seen in the numerical model.

We define key states " $\mathrm{A}$ " and " $\mathrm{B}$ " as in section 2 and take ensemble averages of the PV $\left(\langle q\rangle^{A}\right.$ and $\left.\langle q\rangle^{B}\right)$ and their anomalies. Transects of these quantities through 

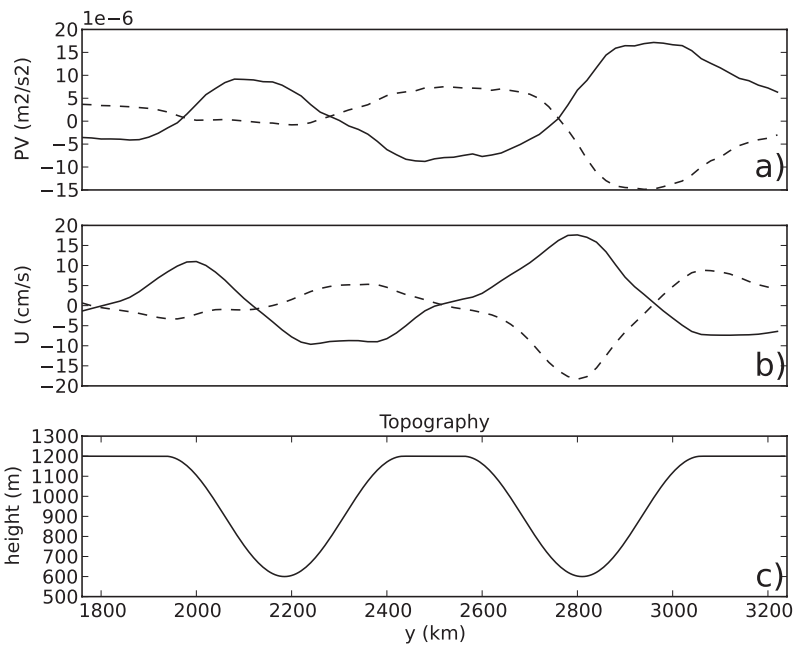

FIG. 11. Transects along the $y$ axis through the center of the domain comparing keys states A (solid) and B (dashed). (a) Ensembleaveraged barotropic PV anomalies $\left(\langle q\rangle^{A}\right.$ and $\left.\langle q\rangle^{B}\right)\left(\mathrm{m}^{2} \mathrm{~s}^{-2}\right)$; (b) ensemble-averaged surface zonal velocity anomalies $\left(\langle u\rangle^{A}\right.$ and $\left.\langle u\rangle^{B}\right)\left(\mathrm{cm} \mathrm{s}^{-1}\right)$; and (c) transect of topography, showing the locations of canyons for reference.

the center of the model domain (which includes the part of the domain where this mode of variability occurs) are displayed in Fig. 11. As predicted by our theory, when the system exists in state A, an enhanced PV gradient (Fig. 11a) is found over the northern canyon while the PV gradient in the vicinity of the southern canyon has reduced substantially. The zonal velocity field reflects these changes in $\mathrm{PV}$, with a stronger jet in the northern canyon and a weaker jet in the southern canyon. The reverse situation is apparent when the system exists in state B.

The principal component time series associated with the first EOF was used in section 2 to describe the time variability of the "jet jumping" behavior, as this PC was highly correlated with transport through the northern canyon and anticorrelated with transport through the southern canyon. Using the low-pass-filtered time series of $\Delta Q^{N}=Q^{N}-Q^{S}$ as a proxy for the strength of the PV staircase, we compare the normalized principal component time series with this metric to assess whether or not differences in the strength of the topographic circulations are able to describe the system's variability. This comparison is shown in Fig. 12, where it is revealed that the two measures of variability exhibit extremely high correlation, lending strong support to the viability of our proposed mechanism. The Pearson $r$ is found to be 0.703 , with a very similar value for the coefficient of determination, and a $p$ value that suggests that the result is significant.

To summarize this section, there is strong evidence from the numerical model that the dynamical mechanism

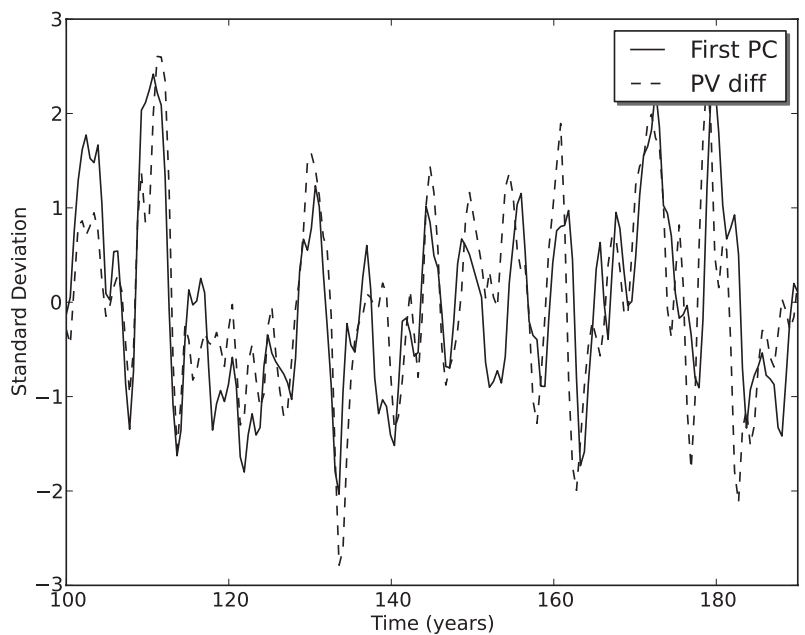

FIG. 12. Comparison of the PC associated with the leading mode of variability (solid) and the difference in integrated PV between the north and south topographic circulations (dashed). Both quantities are centered and normalized by their respective standard deviations.

based on coupled topographic circulations has significant merit. It is clear that the variability in the topographic anticyclones themselves is driven by eddy forcing and that these two circulations show anticorrelated behavior because of the exchange of PV between them by eddy processes. The changing PV contrast between these gyres gives rise to the observed transport variability within the canyons.

\section{d. Are closed $f / H$ contours a necessary condition for low-frequency variability?}

Our proposed mechanism relies on the presence of closed contours of $f / H$. We justify this statement by running an additional computation in which the topographic profile is the same, but the height of the topography $h_{0}$ is reduced such that closed geostrophic contours are no longer formed. ${ }^{3}$ All other parameters are the same as in Table 1. As before, the model is spun up for 70 years. Post spinup, we run the model for an additional 30 years, which we find sufficient for demonstration.

Like the simulation with blocked geostrophic contours, the unblocked simulation shows the development of 3-4 jets that preferentially flow through the canyons. However, it does not show the formation of strong topographic anticyclones. Transport and EOF diagnostics are calculated for this computation as in sections $2 \mathrm{a}$ and 2b. Regarding the former, the time series of transport through each canyon shows time variability but with substantial differences when compared to the situation

\footnotetext{
${ }^{3}$ Because of space constraints, data are not shown.
} 
with blocked geostrophic contours. For example, performing a PC analysis on the two time series, as in section $2 \mathrm{a}$, shows that the anticorrelated mode no longer dominates the correlated mode, explaining $\sim 45 \%$ of the variance, in comparison to $\sim 65 \%$ of the variance in the blocked-contour case. In addition, Fourier analysis no longer reveals the spectral peak at $5 \mathrm{yr}^{-1}$, with the spectrum being dominated by high-frequency variability with frequencies $<1 \mathrm{yr}^{-1}$. Repeating the EOF analysis described in section $2 b$, we do not recover the dipolelike variability evident in the previous simulations. The resulting EOFs are instead dominated by a process without a clear structure, most likely the manifestation of eddy noise. As such, the key-state analysis does not yield clear variations in the strength of the jet through each canyon.

From this analysis, we conclude that without closed $f /$ $H$ contours, the "jet jumping" variability does not manifest itself and thus blocking topography is an essential ingredient in this type of low-frequency variability.

\section{Conclusions and discussion}

Intrinsic, low-frequency variability of jets that interact with a large-scale topographic feature is studied in an idealized, quasigeostrophic channel model. The model is wind forced and the topography consists of a single, meridional ridge with two "canyons." The modeled flow has a strong eddy field and a number of strong eastward jets, reminiscent of the flow in the Southern Ocean. These jets are steered through the canyons in the topography. The low-frequency variability in the model is characterized by jet jumping: large, anticorrelated variations in transport through each of the canyons. In our simulations, the northern canyon can carry between -26 and 203 Sverdrups, while the southern canyon can carry between 35 and 221 Sverdrups. Principal component analysis shows that approximately $65 \%$ of the variance is explained by the anticorrelated mode. We hypothesize that this jet jumping mode of variability is similar to variability observed in satellite-derived data in the vicinity of the Kerguelen Plateau (Sokolov and Rintoul 2009) and in fixed moorings observed through canyons in the Macquarie Ridge, south of New Zealand (Williams et al. 2010). This mode of variability is of interest as fronts in the ACC are strongly associated with sea surface temperature fields, and the shifting of fronts may have important effects on local climate and on the general circulation of the ACC as a whole. We have not attemptedto resolve the quandary about whether hydrographic fronts change their position readily or neighboring fronts simply undergo anticorrelated strengthening or weakening; however, we speculate that the shift of a hydrographic front may be the result of a particularly extreme strengthening/weakening event.

We have proposed a dynamical mechanism to explain this low-frequency variability. This mechanism results from the interplay between closed anticyclonic circulations that form in regions of closed geostrophic contours and the mean background flow. The strength of these circulations is highly variable. This variability is controlled by a balance between PV flux through the closed contours, and frictional damping, as in Venaille et al. (2011). Because of the two neighboring circulations sharing a boundary portion, flux out of one topographic circulation is equal to flux into its neighbor, coupling the neighboring circulations. These topographic circulations form closed "pools" of homogenized potential vorticity. The difference in integrated PV between these two pools of PV is directly related to the strength of the jet that forms between them, by way of the PV staircase.

Comparing the predictions of our dynamical framework with the output from our numerical model, we find good agreement between the two. However, we have neglected to investigate several important components of the system. In particular we have not investigated the effects of the jets acting as transport barriers on the flux of PV between the two topographic circulations; and we have not studied the effects of eddy-mean flow feedback. Given that the strength of jets can dramatically influence both the across-streamline eddy flux and the eddy genesis as a result of baroclinic instability, these effects are likely to be of importance to the time evolution of the system, as they are in the simulations of Hogg and Blundell (2006). In addition, we have not investigated the time scale of the variability in this study.

Despite these shortcomings, this paper demonstrates a relatively simple mechanism of unforced variability in channel flows that may have applicability to the ocean. Future work will concentrate on studying similar mechanisms of variability in satellite altimetry data and more realistic ocean models.

Acknowledgments. The authors would like to acknowledge Prof. W. K. Dewar, Dr. S. R. Rintoul, and Dr. M. Williams for helpful discussions and two anonymous reviewers who greatly improved the paper. Numerical computations were supported by an award under the Merit Allocation Scheme on the National Facility of the Australian Partnership for Advanced Computing. C.C. Chapman is supported by a CSIRO Wealth From Oceans Flagship Scholarship.

\section{REFERENCES}

Andrews, D. G., and M. E. McIntyre, 1976: Planetary waves in horizontal and vertical shear: The generalized Eliassen-Palm 
relation and the mean zonal acceleration. J. Atmos. Sci., 33, 2031-2048.

Berloff, P., A. M. C. Hogg, and W. Dewar, 2007: The turbulent oscillator: A mechanism of low-frequency variability of the wind-driven ocean gyres. J. Phys. Oceanogr., 37, 2363-2386.

Dewar, W. K., 1998: Topography and barotropic transport control by bottom friction. J. Mar. Res., 56, 295-328.

Dritschel, D. G., and M. E. McIntyre, 2008: Multiple jets as PV staircases: The Phillips effect and the resilience of eddy transport barriers. J. Atmos. Sci., 65, 855-874.

Dunkerton, T. J., and R. K. Scott, 2008: A barotropic model of the angular momentum-conserving potential vorticity staircase in spherical geometry. J. Atmos. Sci., 65, 1105-1136.

Hogg, A. M., and J. R. Blundell, 2006: Interdecadal variability of the Southern Ocean. J. Phys. Oceanogr., 36, 1626-1645.

—, W. K. Dewar, P. D. Killworth, and J. R. Blundell, 2003: A quasigeostrophic coupled model (q-GCM). Mon. Wea. Rev., 131, 2261-2278.

Plumb, R. A., and R. Ferrari, 2005: Transformed Eulerian-mean theory. Part I: Nonquasigeostrophic theory for eddies on a zonal-mean flow. J. Phys. Oceanogr., 35, 165-174.

Rintoul, S. R., C. W. Hughes, and D. Olbers, 2001: The antarctic circumpolar current system. Ocean Circulation and Climate, G. Siedler, J. Chruch, and J. Gould, Eds., Academic Press, 271-302.

Sallée, J. B., K. Speer, and R. Morrow, 2008: Response of the Antarctic circumpolar current to atmospheric variability. J. Climate, 21, 3020-3039.

Saunders, P. M., and B. A. King, 1995: Bottom currents derived from a shipborne ADCP on WOCE cruise A11 in the South Atlantic. J. Phys. Oceanogr., 25, 329-347.

Sokolov, S., and S. R. Rintoul, 2009: Circumpolar structure and distribution of the Antarctic circumpolar current fronts: 2.
Variability and relationship to sea surface height. J. Geophys. Res., 114, C11019, doi:10.1029/2008JC005248.

Thompson, A. F., 2010: Jet formation and evolution in baroclinic turbulence with simple topography. J. Phys. Oceanogr., 40, 257-278.

— , P. H. Haynes, C. Wilson, and K. J. Richards, 2010: Rapid southern ocean front transitions in an eddy-resolving ocean GCM. Geophys. Res. Lett., 37, L23602, doi:10.1029/ 2010 GL045386.

Vallis, G. K., and M. E. Maltrud, 1993: Generation of mean flows and jets on a beta plane and over topography. J. Phys. Oceanogr., 23, 1346-1362.

_ , E. P. Gerber, P. J. Kushner, and B. A. Cash, 2004: A mechanism and simple dynamical model of the North Atlantic oscillation and annular modes. J. Atmos. Sci., 61, 264-280.

Venaille, A., 2012: Bottom-trapped currents as statistical equilibrium states above topographic anomalies. J. Fluid Mech., 699, $500-510$

_- J. Le Sommer, J. M. Molines, and B. Barnier, 2011: Stochastic variability of oceanic flows above topography anomalies. Geophys. Res. Lett., 38, L16611, doi:10.1029/2011GL048401.

Volkov, D. L., and L.-L. Fu, 2008: The role of vorticity fluxes in the dynamics of the Zapiola anticyclone. J. Geophys. Res., 113, C11015, doi:10.1029/2008JC004841.

Wilks, D. S., 2006: Statistical Methods in the Atmospheric Sciences. 2nd ed. International Geophysics Series, Vol. 91 Academic Press, $648 \mathrm{pp}$

Williams, M. J., S. Sokolov, S. R. Rintoul, and N. Bindoff, 2010: Temporal variability in Antarctic circumpolar current transport across the Macquarie Ridge. Proc. Int. Polar Year Oslo Science Conf., Oslo, Norway, International Polar Year, T1-1.

Williams, P. D., 2009: A proposed modification to the RobertAsselin time filter. Mon. Wea. Rev., 137, 2538-2546. 